\title{
Verfahren zur Bestimmung von Materialkonstanten zweier aneinandergrenzender Gesteinsschichten auf reflexionsseismischem Wege
}

\author{
E. HaRdTwig $(*)$
}

Received on May 20 th, 1977.

Riassunto - Il presente articolo indica la possibilità di determinare le costanti materiali di due strati a contatto fra loro lungo un piano. A tale scopo si utilizza l'andamento, da determinarsi sperimentalmente, dei due coefficienti di riflessione $C / A$ e $D / A$ in funzione dell'angolo di incidenza di un'onda di compressione. Nell'intervallo $0^{\circ}-80^{\circ}$ di tale angolo i coefficienti di riflessione presentano un andamento talmente differenziato da poterne dedurre delle equazioni di condizione per le costanti materiali, che rendono possibile il calcolo di queste ultime. Si eseguono i calcoli per un "caso tipico» tratto dalla pratica, e ne vengano costruite le curve. Dato che $\mathrm{i}$ casi che si presentano in pratica sono relativamente poco diversi dal "caso tipico", questo può venire utilizzato a scopo orientativo. Non vengono qui date indicazioni sul modo di determinare sperimen. talmente i coefficienti di riflessione.

Zusammenfassung - Der vorliegende Aufsatz weist auf die Möglichkeit hin, die Materialkonstanten zweier, entlang einer Ebene aneinandergrenzenden Schichten zu bestimmen. Dazu wird der experimentell zu bestimmende Verlauf der beiden Reflexionskoeffizienien $C / A$ und $D ; A$ als Funktionen des Einfallswinkels einer Verdichtungswelle benutzt. Im Bereich zwischen 0 und $80^{\circ}$ dieses Winkels zeigen die Reflexionskoeffizienten ein so abwechslungsreiches Verhalten, dass daraus Bedingungsgleichungen für die Materialkonstanten abgeleitet werden können, die ihre Berechnung möglich machen. An einem der Praxis entnommenen "Musterfall" werden die Rechnungen durchgeführt und die Kurven gezeichnet. $\mathrm{Da}$ die in der Praxis vorkommenden Fälle verhältnismassig wenig vom: Musterfall abweichen, kann dieser als Orientierungshilfe benutzt werden. Hinweise darauf, wie die Reflexionskoeffizienten tatsächlich durch Messung bestimmt werden können, werden nicht gegeben.

$\left(^{*}\right)$ Il presente lavoro è pubblicato postumo. Le bozze di stampa sono state corrette dalla Redazione della Rivista. 
Die Reflexionsseismik hat bekanntlich die Aufgabe, die Tiefe einer geologischen Unstetigkeitstläche (Schichtgrenze) aus der Laufzeit solcher Wellenimpulse zu bestimmen, die, von einem oberflächennahen Sprengpunkt ausgehend, nach ihrer Reflexion an der Unstetigkeitsfläche, wieder an die Erdoberfläche zurückkehren. Die Wellengeschwindigkeit wird dabei als bekannt vorausgesetzt.

Es kommt bei diesen Verfahren nur auf die Laufzeiten der Reflexionen an, also auf die Festellung der Einsätze im Seismogram. Die gelegentlich recht teueren Sprengschüsse dienen also nur dazu, solche Reflexionen $\mathrm{zu}$ liefern. Sonstige Aufschlüsse über die Beschaffenheit des Erdinnern, genauer: über die physikalischen Eigenschaften der beiden Schichten, die entlang der Reflexionsfläche aneinandergrenzen, liefert dieses Verfahren nicht. Es fragt sich, ob tatsächlich alle Möglichkeiten ausgeschöpft sind, durch einen Schuss Aufschlüsse über das Erdinnere - im obgenannten Sinne - zu erhalten, mit anderen Worten, ob der Schuss nach dem bisherigen Verfahren der Reflexionsseismik rationell ausgenutzt wird. Die Antwort kann nur verneinend sein. Die Theorie der Reflexion und Brechung seismischer Wellen an Unstetigkeitsflächen zeigt, dass erstens in das Problem einige wichtige, auf die beiden aneinandergrenzenden Schichten bezügliche und für sie charakteristische physikalische Konstanten eingehen und dass zweitens die grundsätzliche Möglichkeit besteht, diese Konstanten durch Beobachtungen an der Erdoberfläche zu bestimmen.

Das dabei einzuschlagende Verfahren wird im folgenden beschrieben. Es bedient sich der Messung der Reflexionskoeffizienten und ihrer Darstellung als Funktion des Einfallswinkels der Primärwelle in Bereichen dieses Winkels, in dem sie besonders abwechslungsreich und charakteristisch verlaufen.

1.

Die Theorie der Brechung und Reflexion seismischer Wellen an ebenen Unstetigkeitsflächen darf im wesentlichen als bekannt vorausgesetzt werden. Sie wurde 1899 von C. G. Knott begründet und später von $\mathrm{H}$. Blut und anderen weiterentwickelt. Hier soll nur, an ihre Grundzüge erinnert werden.

Beim Auftreffen einer ebenen Welle auf die Unstetigkeitsebene - wir nehmen als einfallende Welle eine Verdichtungswelle ( $P$-Welle) an - hört 
diese zu existieren auf, dafür entstehen vier Sekundärwellen, nämlich je eine gebrochene und reflektierte Verdichtungs - und Scherungswelle. Fig. I gibt die Verhältnisse unter den unten genannten Voraussetzungen qualitativ wieder. Es wurde bezeichnet

(a) Einfallende Verdichtungswelle

(c) Rellektierte

Verdichtungswelle

(e) Gebrochene

(d) Reflektierte

Scherungswelle

(f) Gebrochene.

Die beiden aneinandergrenzenden, als voll elastische Körper angenommenen Schichten wurden mit Schicht 1 (oben) und Schicht 2 (unten) bezeichnet.

Weitere Bezeichnungen:

$\rho_{1}, \rho_{2}$ Dichte von Medium 1 bzw. 2;

$V_{1}, V_{2}$ Phasengeschwindigkeit der Verdichtungswelle in Medium 1 bzw. 2:

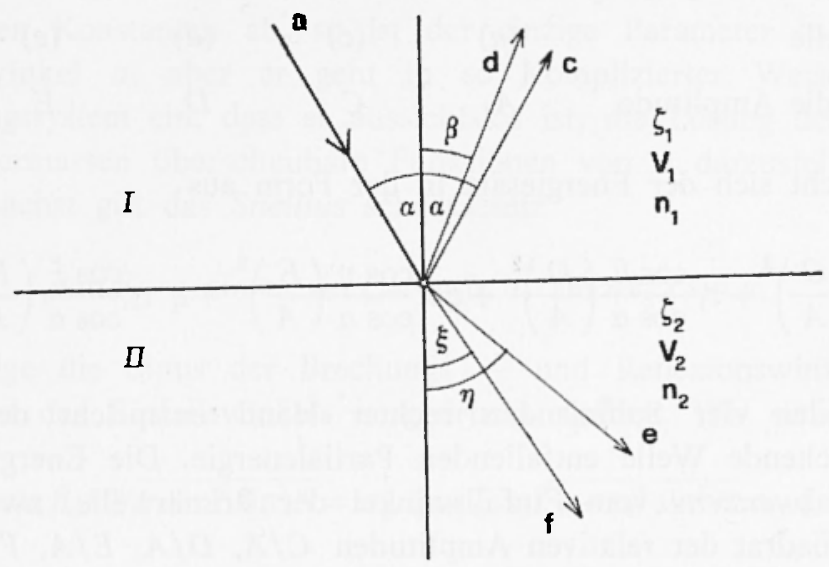

Fig. 1. 
$n_{1}, n_{2}$ Phasengeschwindigkeit der Scherungswelle in Medium i bzw. 2;

$$
q=\frac{\rho_{2}}{\rho_{1}}, x=\frac{V_{2}}{V_{1}} . \tau_{1}=\frac{n_{1}}{V_{1}}, \tau_{2}=\frac{n_{2}}{V_{1}}
$$

Wir setzen voraus

Dann ist

$$
\tau_{1}<1, x>1, \tau_{2}>1
$$

$$
\eta>\xi>\alpha>\beta
$$

und es findet Brechung vom Lot weg statt, wie in Fig. 1 angedeutet. Totalreflexion ist sowohl hinsichtlich der (e)-Welle wie hinsichtlich der $(f)$-Welle möglich, wenn nur der Einfallswinkel $\alpha$ der Primärwelle gross genug gewählt wird.

Die Energie, die von der einfallenden Welle bis an die Unstetigkeitsfläche gebracht wurde, verschwindet, dafür bekommt jede der neuenststandenen Sekundärwellen ein Energiequantum mit auf den Weg, aber in solcher Weise, dass die Summe der ausstrahlenden Energien gleich ist der eingestrahlten Energie. Wählt man den Massstab so, dass die eingestrahlte Energie den Wert Eins hat und bezeichnet man die Amplituden der einzelnen Wellen entsprechend dem Schema

zur Welle

gehört die Amplitude (a)

$A$ (c)

$C$ (d)

$D$ (e)

E $(f)$

$F$,

so spricht sich der Energiesatz in der Form aus

$$
1=\left(\frac{C}{A}\right)^{2}+\tau_{1} \frac{\cos \beta}{\cos \alpha}\left(\frac{D}{A}\right)^{2}+q_{x} x \frac{\cos \eta}{\cos \alpha}\left(\frac{E}{A}\right)^{2}+q \tau_{2} \frac{\cos \xi}{\cos a}\left(\frac{F}{A}\right)
$$

Jeder der vier Summanden rechter Hand entspricht der auf die entsprechende Welle entfallenden Partialenergie. Die Energieverteilung hängt ab erstens vom Einfallswinkel der Primärwelle, zweitens aber vom Quadrat der relativen Amplituden $C / A, D / A, E / A, F / A$.

Man nennt $C / A$ und $D / A$ die Reflexionskoeffizienten, $E / A$ und $F / A$ die Transmissionskoeflizienten. 
Sie sind Funktionen des Einfallswinkels $\alpha$ der Primärwelle und müssen, wie hier als bekannt vorausgesetzt werden darf, dem linearen Gleichungssystem

(1) $-\left(\frac{C}{A}\right)+\frac{\sin \hat{\beta}}{\cos \frac{D}{u}}\left(\frac{D}{A}\right)-\frac{\cos r_{1}}{\cos \alpha}\left(\frac{E}{A}\right)-\frac{\sin \xi}{\cos \alpha}\left(\frac{F}{A}\right)=-1$

(2) $+\left(\frac{C}{A}\right)+\frac{\cos \beta}{\sin \alpha}\left(\frac{D}{A}\right)-\frac{\sin r_{1}}{\sin \alpha}\left(\frac{E}{A}\right)+\frac{\cos \xi}{\sin \alpha}\left(\frac{F}{A}\right)=-1$

(3) $-\left(\frac{C}{A}\right)+\tau_{1} \frac{\sin 2 \beta}{\cos 2 \beta}\left(\frac{D}{A}\right)+q x \frac{\cos 2 \eta}{\cos 2 \beta}\left(\frac{E}{A}\right)+q \tau_{2} \frac{\sin 2 \xi}{\cos 2 \beta}\left(\frac{F}{A}\right)=1$

(4) $+\left(\frac{\iota}{A}\right)+\frac{1}{\tau_{1}} \frac{\cos 2 \hat{j}}{\sin 2 \alpha}\left(\frac{\bar{\nu}}{A}\right)+q \frac{\tau_{2}^{2}}{\tau_{1}^{2} x} \frac{\sin 2 \eta}{\sin 2 \alpha}\left(\frac{E}{A}\right)-$

$$
-q \frac{\tau_{2}}{\tau_{1}^{2}} \frac{\cos 2 \xi}{\sin 2 \alpha}\left(-\frac{F}{A}\right)=1
$$

Da die Determinante des Systems nich indentisch verschwindet, gibt es eine von Null verschiedene Lösung $C / A, D / A, E / A, F / A$.

2. Sieht man von den in den Grössen $q, \tau_{1}, \tau_{2}$ enthaltenen physikalischen Konstanten $a b$, so ist der einzige Parameter in [5] der Einfallswinkel $\alpha$, aber er geht in so Komplizierter Weise in das Gleichungssystem ein, dass es aussichtslos ist, die Lösung des Systems als einigermassen überschaubare Funktionen von $\alpha$ darzustellen.

Zunächst gilt das Snellius' sche Gesetz

$$
\sin \beta=\tau_{1} \sin \alpha, \sin \xi=\tau_{2} \sin \alpha, \sin \eta=x \sin \alpha,
$$

demzufolge die Sinus der Brechungs - und Reflexionswinkel durch den Sinus des Einfallswinkels ausgedrückt werden. Wir setzen

$$
\begin{gathered}
\cos \beta=\sqrt{1-\tau_{1}^{2} \sin ^{2} \alpha}=w_{1}, \cos \xi=\sqrt{1-\tau_{2}^{2} \overline{\sin ^{2} \alpha}}=w_{2}, \\
\cos \eta=\sqrt{1-x^{2} \sin ^{2} \alpha}=w
\end{gathered}
$$


und können die Energiegleichung in folgender Weise schreiben:

$$
1=\left(\frac{C}{A}\right)^{2}+\tau_{1} \frac{w_{1}}{\cos \alpha}\left(\frac{D}{A}\right)^{2}+q \varkappa \frac{w}{\cos \alpha}\left(\frac{E}{A}\right)+q \tau_{2}-\frac{w_{2}}{\cos \alpha}\left(\frac{F}{A}\right)^{2} .
$$

Wollte man auch in System [5] alle auftretenden Winkelfunktionen durch solche des Winkels $\alpha$ ersetzen, so würde das System für die rechnerische Behandlung ausserordentlich unhandlich werden. Erst durch Verwendung eines Computers lassen sich dann die Koeffizienten bei vorgegebenen $\alpha$-Werten zahlenmässig in kürzester Zeit bestimmen und das System auflösen. Dabei verzichtet man naturgemäss auf die allgemeine Darstellung der Reflexions - und Transmissionskoeffizienten als Funktionen des Einfallswinkels. Für das Durchrechnen von im Gelände auftretenden konkreten Fällen ist es zweckmässig, einen Musterfall vor Augen zu haben, von dem diese Fälle im allgemeinen nur wenig abweichen.

Als Orientierungshilfe soll folgender spezielle, aber in der Natur realisierte Fall angenommen werden:

$$
\begin{aligned}
& \frac{\rho_{2}}{\rho_{1}}=a=1,2 ; \quad \frac{V_{2}}{V_{1}}=x=\frac{3500 \mathrm{~m} / \mathrm{s}}{1800 \mathrm{~m} / \mathrm{s}}=1,9 \overline{4} ; \\
& \tau_{1}=\frac{n_{1}}{V_{1}}=\frac{V \overline{3}}{3}=0,5774 ; \quad \tau_{2}=\frac{\sqrt{3}}{5} x=1,1226 .
\end{aligned}
$$

Das System [5] sieht dann folgendermassen aus

(1) $-\left(\frac{C}{A}\right)+0.5774 \cdot \operatorname{tg} \alpha\left(\frac{D}{A}\right)-\frac{\cos \eta}{\cos \alpha}\left(\frac{E}{A}\right)-1.1226 \operatorname{tg} \alpha\left(\frac{F}{A}\right)=-1$

(2) $+\left(\frac{C}{A}\right)+0.5774 \cdot \operatorname{ctg} \beta\left(\frac{D}{A}\right)-1.94 \cdot\left(\frac{E}{A}\right)+1.1226 \cdot \operatorname{ctg} \xi\left(\frac{F}{A}\right)=-1$

(3) $-\left(\frac{C}{A}\right)+0.5774 \cdot \operatorname{tg} 2 \beta\left(\frac{D}{A}\right)+2.3 \frac{\cos 2 \eta}{\cos 2 \beta}\left(\frac{E}{A}\right)$

$$
+1.3472 \frac{\sin 2 \xi}{\cos 2 \beta}\left(\frac{F}{A}\right)=+1
$$

(4) $+\left(\frac{C}{A}\right)+1.7321 \cdot \frac{\cos 2 \beta}{\sin 2 \alpha}\left(\frac{D}{A}\right)+2.3 \cdot \frac{\sin 2 \eta}{\sin 2 \alpha}\left(\frac{E}{A}\right)-4.0414 \frac{\cos 2 \xi}{\sin 2 \alpha}=1$. 
Hier ist implizit die Annahme $\sigma=1 / 4$ für die Poissonsche Konstante enthalten. Der Einfallswinkel $\alpha$ bleibt variabel, sein Variabilitätsbereich erstreckt sich von Null bis unmittelbar vor den Grenzwinkel der ersten Totalreflexion. Dieser ist bekanntlich dadurch charakterisiert, dass die gebrochene sekundäre Verdichtungswelle (e) sich dem streifenden Austritt nähert, für den

$$
\eta=90^{\circ}, \sin \eta=1, \cos \eta=0
$$

ist. Wir haben also

$$
x \cdot \sin \alpha_{0}=1 \text {, und mithin } \sin \alpha_{0}=1 / x=V_{1} / V_{2} \text {. }
$$

In unserem Musterffale ist der Grenzwinkel der Totalreflexion (der Machsche Winkel) also $\alpha_{0}=30^{\circ} 57^{\prime} 02^{\prime \prime}$. Das Gleichungssystem [10] des Musterfalles wurde streng für die folgenden $\alpha$-Werte aufgelöst:

$$
\alpha=0^{0}, 10^{\circ}, 20^{\circ}, 30^{\circ}, 30^{\circ} 57^{\prime} 02^{\prime \prime} \mathrm{Grad} .
$$

Derr Wert von $C / A$ für den Machschen Winkel gilt nur formal, für ihn tritt Totalreflexion ein, die durch das System [5] bzw. [10] nicht beschrieben wird.

Das Ergebnis der Rechnung zeigt die folgende Tabelle 1

TABELLE 1

\begin{tabular}{rllll}
\hline$\alpha$ & $C / A$ & $D / A$ & $E / A$ & $F / A$ \\
\hline 0 & 0.4 & 0, & 0,6 & 0, \\
10 & 0,3808 & $-0,1497$ & 0,6077 & 0,1166 \\
20 & 0.3551 & $-0,2505$ & 0,6477 & 0.2377 \\
30 & 0,4931 & $-0,0719$ & 0,9828 & 0,3354 \\
\hline$\alpha^{0}$ & $(0,92507)$ & $+0,29440$ & 1,48568 & 0,26271 \\
\hline
\end{tabular}

Man erkennt ein sehr starkes Ansteingen der beiden Reflexionskoeffizienten $C / A$ und $D / A$ im Bereich des Machschen Winkels. 
Wächst der Einfallswinkel über den Machschen Winkel hinaus, so wird $x \sin \alpha>1$ und das Symbol $\sin \eta$ ist nur eine Abkürzung für den Ausdruck $\varkappa \sin \alpha$. Gemäss

$$
\cos \eta=\sqrt{1-\sin ^{2} \eta}=\sqrt{1-\varkappa^{2} \sin ^{2} \alpha}= \pm i \sqrt{\varkappa^{2} \sin ^{2} \alpha-1}= \pm i w^{*}
$$

wird $\cos \eta$ imaginär.

Die Wahl des Vorzeichens für die Wurzel ist an und für sich willkürlich. Der Einfachheit halber soll das + Zeichen gewählt werden, wir behalten uns jedoch vor, später, wenn $\cos \eta$ im Exponenten einer reellen $e$-Potenz auftritt, das Minuszeichen zu wählen, um ein Wachsen des Ausdrucks ins Unendliche bei wachsender Entfernung von der Unstetigkeitsfläche $\mathrm{zu}$ vermeiden.

Das System [5] zur Bestimmung der Amplitudenverhältnisse bekommt nun folgendes Aussehen:

$$
\begin{aligned}
& -\left(\frac{C}{A}\right)+\tau_{1} \operatorname{tg} \alpha\left(\frac{D}{A}\right)-i \frac{w^{*}}{\cos \alpha}\left(\frac{E}{A}\right)-\tau_{2} \operatorname{tg} \alpha\left(\frac{F}{A}\right)=-1 \\
& +\left(\frac{C}{A}\right)+\frac{\cos \beta}{\sin \alpha}\left(\frac{L^{\prime}}{A}\right)-x\left(\frac{E}{A}\right)+\frac{\cos \xi}{\sin \alpha}\left(\frac{F}{A}\right)=-1 \\
& -\left(\frac{C}{A}\right)+\tau_{1} \frac{\sin 2 \beta}{\cos 2 \beta}\left(\frac{D}{A}\right)+\dot{y} \pi \frac{\cos 2 \eta}{\cos 2 \beta}\left(\frac{E}{A}\right)+\tau^{-} \tau_{2} \frac{\sin 2 \xi}{\cos 2 \beta}\left(\frac{F}{A}\right)=1 \\
& +\left(\frac{C}{A}\right)+\frac{1}{\tau_{1}} \frac{\cos 2 \beta}{\sin 2 \alpha}\left(\frac{D}{A}\right)+i q x^{2} \frac{w^{*}}{\cos \alpha}\left(\frac{E}{A}\right)-q \frac{\tau_{2}}{\tau_{1}^{2}} \frac{\cos 2 \xi}{\sin 2 \alpha}\left(\frac{F}{A}\right)=1
\end{aligned}
$$

Das Auftreten der imaginären Einheit $i$ bewirkt, dass nun alle Amplitudenverhältnisse complex werden.

$$
\text { Man setz }
$$

$$
\begin{array}{ll}
C / A=C^{\prime}+i C^{\prime \prime} & D / A=D^{\prime}+i D^{\prime \prime} \\
E / A=E^{\prime}+i E^{\prime \prime} & F / A=F^{\prime}+i F^{\prime \prime},
\end{array}
$$

wo nun die gestrichenen Grössen reell sind.

Wählt man in [11] das untere Vorzeichen der Wurzel, so geht $i$ über in $-i$ und die komplexen Zahlen [13] gehen in die konjugiert- 
komplexen Zahlen über. Physikalisch ist dies belanglos, da bei den Amplituden der Wellen, aber auch bei den Reflexions - und Transmissionskoeflizienten jeweils der absolute Betrag massgebend ist, nicht die komplexe Zahl als solche.

Man wird mehr order weniger zwangsläufig dazu geführt, die Beträge der komplexen Zahlen für die entsprechenden Amplituden zu nehmen, was von vornherein durchaus nicht selbstverständilich ist. Der Vorteil besteht darin, dass man das System [5] auch dann weiterbehalten kann, wenn in ihm imaginäre Grössen auftreten.

Als Beispiel möge wieder der Musterfall, und zwar für $\alpha={ }^{0} 40^{\prime}$ herangezogen werden. Das System [12] lautet jetzt

$$
\begin{aligned}
& -\left(\frac{C}{A}\right)+0,48445\left(\frac{D}{A}\right)-0,97879 i\left(\frac{E}{A}\right)-0,94199\left(\frac{F}{A}\right)=-1 \\
& +\left(\frac{C}{A}\right)+1,44465\left(\frac{D}{A}\right)-1,94\left(\frac{E}{A}\right)+1,07703\left(\frac{F}{A}\right)=-1 \\
& -\left(\frac{C}{A}\right)+0,54920\left(\frac{D}{A}\right)-0,13345\left(\frac{E}{A}\right)+1,85772\left(\frac{F}{A}\right)=+1 \\
& +\left(\frac{C}{A}\right)+1,27432\left(\frac{D}{A}\right)+4,44077 i\left(\frac{E}{A}\right)+0,17006\left(\frac{F}{A}\right)=+1
\end{aligned}
$$

Seine Auflösung ergibt

$$
\begin{aligned}
& C / A=-0,31992+0,28269 i, D / A=-0,66481-0,37598 i \\
& E / A=0,17440-0,46598 i, F / A=0,57515-0,07449 i
\end{aligned}
$$

Dic Beträge für die Reflexions - und Transmissions koeffizienten sind sind

$$
\begin{aligned}
& \frac{C}{A}=\sqrt{C^{\prime 2}+C^{\prime \prime 2}}, \quad \frac{D}{A}=\sqrt{D^{\prime 2}+D^{\prime \prime 2}}, \\
& \frac{E}{A}=\sqrt{E^{\prime 2}+E^{\prime \prime 2}}, \quad \frac{F}{A}=\sqrt{F^{\prime 2}+F^{\prime \prime 2}},
\end{aligned}
$$




\section{Tabelle II}

Amplitudenverhältnisse der Sekundärwellen.

\begin{tabular}{lcccc}
\hline & $C / A$ & $D / A$ & $E / A$ & $F / A$ \\
\hline$\alpha_{0}$ & $(+0.92507)$ & +0.29440 & 1.48568 & 0.26271 \\
\hline $52^{0}$ & $+0.55425+0.58395 i$ & $+0.03538+0.48875 i$ & $+1.12520+0.66517 i$ & $0.37584-0.11119 i$ \\
35 & $-0.02934+0.56655 i$ & $-0.41493+0.55946 i$ & $+0.51225+0.73713 i$ & $0.53075-0.04017 i$ \\
40 & $-0.31992+0.28269 i$ & $-0.66481+0.37598 i$ & $+0.17440+0.46598 i$ & $0.57515+0.07449 i$ \\
50 & $-0.48467+0.04365 i$ & $-0.68649+0.13581 i$ & $+0.04980+0.13897 i$ & $0.57827+0.10120 i$ \\
60 & $-0.67413+0.00495 i$ & $-0.54228-0.04877 i$ & $-0.02437-0.03109 i$ & $0.62536-0.06154 i$ \\
\hline$\alpha_{1}$ & $-0.80925+0.09813 i$ & $-0.49342-0.25383 i$ & $-0.14621-0.07521 i$ & $(+0.67475-0.38190 i)$ \\
\hline $64^{0}$ & $-0.91618-0.00587 i$ & $-0.29083-0.23934 i$ & $-0.12205-0.19464 i$ & $0.95940-0.29389 i$ \\
65 & $-0.95063-0.07218 i$ & $-0.19632-0.19926 i$ & $-0.09109-0.24637 i$ & $1.06065-0.20240 i$ \\
70 & $-0.95519-0.16019 i$ & $-0.19659-0.04123 i$ & $-0.11582-0.36840 i$ & $1.06439-0.21687 i$ \\
80 & $-0.92302-0.24595 i$ & $+0.02725+0.17680 i$ & $+0.13075-0.20170 i$ & $0.61400+0.32269 i$ \\
\hline
\end{tabular}


Das Vorzeichen der Wurzeln wird durch Stetigkeitsüberlegungen bestimmt.

Im Musterfall sind zufolge [16] die Beträge von $C / A, D / A, F / A$ gegeben durch

$$
\left|\frac{C}{A}\right|^{2}=0,18225 ;\left|\frac{D}{A}\right|^{2}=0,58332 ;\left|\frac{F}{A}\right|^{2}==0,33634 \text {. }
$$

Diese Zahlen werden bei der Verifizierung der Energiegleichung in dem speziellen Fall $\alpha=40^{\circ}$ benötigt.

Wie für eine Reihe von Werten des Einfallswinkels die Amplitudenverhältnisse aussehen, zeigt Tabelle II. Sie wurden aus dem System [14] auf fünf Dezimalen genau gerechnet.

3.

Die Energiegleichung [4] wurde bisher beiseite gelassen. Man kann aber versuchen, sie auf den Fall zu übertragen, dass der Grenzwinkel der totalen Reflexion überschritten wird. Mathematisch gesehen stellt sie eine Identität dar - dieser Charakter wird offenbar, wenn man für die Verhältnisse $C / A, D / A \ldots$ jene Ausdrücke einsetzt, die sich durch Auflösen des Linearsystems [12] ergeben.

An diesem Sachverhalt kann sich nichts ändern, wenn der Winkel $\eta$ den Betrag von $90^{9}$ erreich oder wenn $\cos \eta$ imaginar wird. Dei Frage ist nur, ob die Gleichung auch dann noch als Energiegleichung gedeutet werden darf.

Wir nehmen dies an, überzeugen uns aber an Hand eines Zahlenbeispiels, ob diese Deutung sinnvoll ist.

Wenn die Gleichung [4] auch für den hier vorliegenden Fall weiterbesteht darf man, ohne ihre Richtigkeit zu zerstören, für $C / A$, $D / A, \ldots$ die entsprechenden complexen Zahlen einsetzen:

$$
\begin{aligned}
\left(C^{\prime}+i C^{\prime \prime}\right)^{2}+\tau_{1} \frac{\operatorname{c\Omega s} \frac{R}{\cos \alpha}}{\cos \alpha}\left(D^{\prime}+i D^{\prime \prime}\right)^{2} & +q \tau_{2} \frac{\cos \xi}{\cos \alpha}\left(F^{\prime}+i F^{\prime \prime}\right)^{2}+ \\
& +i q x \frac{w^{*}}{\cos \alpha}\left(E^{\prime}+i E^{\prime \prime}\right)^{2}=1 .
\end{aligned}
$$

Ordnet man nach Real- und Imaginärteil, so bleibt 


$$
\begin{aligned}
& \left(C^{\prime 2}-C^{\prime \prime 2}\right)+\tau_{1} \frac{2 \Omega \varepsilon R}{\cos \alpha}\left(D^{\prime \prime}-D^{\prime \prime 2}\right)+ \\
& q \tau_{2}+\frac{\cos \xi}{\cos \alpha}\left(F^{\prime 2}-F^{\prime \prime 2}\right)-2 q x \frac{w^{*}}{\cos \alpha}\left(E^{\prime} E^{\prime \prime}\right)+ \\
& +i\left[2 C^{\prime} C^{\prime \prime}+2 \tau_{1} \frac{\cos \beta}{\cos \alpha} D^{\prime} D^{\prime \prime}+2 q \tau_{2} \frac{\frac{2 n \varepsilon}{2}}{\cos \alpha} F^{\prime} F^{\prime \prime}+\right. \\
& \left.+q \times \frac{1, \cdots *}{\cos \alpha}\left(E^{\prime 2}-E^{\prime \prime 2}\right)\right]=1 \text {. }
\end{aligned}
$$

Der Realteil ergibt die Gleichung

$$
\begin{gathered}
\left(C^{\prime 2}-C^{\prime \prime 2}\right)+\tau_{1} \frac{\cos \beta}{\cos \alpha}\left(D^{\prime 2}-D^{\prime \prime 2}\right)+q \tau_{2} \frac{\frac{2 n \varepsilon}{\cos \alpha}}{\cos }\left(F^{\prime 2}-F^{\prime 2}\right)- \\
-2 q x \frac{w^{*}}{\cos \alpha}\left(E^{\prime} E^{\prime \prime}\right)=1
\end{gathered}
$$

und der Imaginärteil muss für sich verschwinden:

$$
\begin{aligned}
2\left(C^{\prime} C^{\prime \prime}+\tau_{1} \frac{\cos \beta}{\cos \alpha} D^{\prime} D^{\prime \prime}+q \tau_{2}\right. & \left.\frac{\cos \xi}{\cos \alpha} F^{\prime} F^{\prime \prime}\right)+ \\
& +q x \frac{w^{\prime}}{\cos \alpha}\left(E^{\prime 2}-E^{\prime \prime}\right)=0
\end{aligned}
$$

Gleichung [18] lässt sich in dieser Form physikalisch nich deuten, wohl aber, wenn man sie durch Hinzufügen und Wegnehmen des Ausdruckes

$$
2\left(C^{\prime \prime 2}+\tau_{1} \frac{\cos \beta}{\cos \alpha} D^{\prime \prime 2}+q \tau_{2} \frac{\cos \xi}{\cos \alpha} F^{\prime \prime 2}\right)
$$

umformt. Dann lautet sie nämlich im Hinblick auf [16]

$$
\begin{aligned}
\left|\frac{C}{A}\right|^{2}+ & \tau_{1} \frac{\cos \beta}{\cos \alpha}\left|\frac{D}{A}\right|^{2}+q \tau_{2} \frac{\cos \xi}{\cos \alpha}\left|\frac{F}{A}\right|^{2}-2 q x \frac{w^{\prime \prime}}{\cos \alpha} E^{\prime} E^{\prime \prime}- \\
& -2\left(C^{\prime \prime 2}+\tau_{1} \frac{\cos \beta}{\cos \alpha} D^{\prime \prime 2}+q \tau_{2} \frac{\cos \xi}{\cos \alpha} F^{\prime \prime 2}\right)=1
\end{aligned}
$$


und in dieser Form lässt sie die Deutung als Energiegleichung zu: wenn nämlich bedeutet

$$
\left|\frac{C}{A}\right|^{2} \text { die Energie } J_{c} \text { der reflektierten } P \text {-Welle, }
$$

$\tau_{1} \frac{\cos \beta}{\cos \alpha}\left|\frac{D}{A}\right|^{2}$ die Energie $J_{d}$ der reflektierten $S$-Welle,

$q \tau_{2} \frac{\cos \xi}{\cos \alpha}\left|\frac{F}{A}\right|^{2}$ die Energie $I_{f}$ der gebrochenen $S$-Welle,

so müssen notwendig die restlichen Glieder auf der linken Seite von [21] die Energie der die Unstetigkeitstläche entlang geführten Welle bedeuten (die an die Stelle der gebrochenen $P$-Welle tritt). Auch dieser Energiebetrag muss positiv sein, obwohl formell alle Summanden das Minuszeichen haben. Tatsächlich ist aber das erste Glied positiv, denn $E^{\prime}$ und $E^{\prime \prime}$ sind entgegengesetzt bezeichne; ausserdem ist dieses Glied dem Betrage nach grösser als jedes der übrigen Glieder.

Die der Unstetigkeitsfläche entlang geführte Welle ist, wenn sie überhaupt Energie mit sich führt, sehr energiearm, wie spezielle Beispiele zeigen. Sie verhält sich wie eine Wellenhaut und hat ein Analogon in der Optik. Wegen der Kompliziertheit der Ausdrücke für $C^{\prime}, C^{\prime \prime}, D^{\prime}, D^{\prime \prime}, \ldots$ dürfte sich dies nur mühsam beweisen lassen, leichter schon das Bestehen von Gl. [19], die das Verschwinden des Imaginärteils der Energiegleichung aussagt. Für den hier vorliegenden $Z$ weck genügt es, die Richtigkeit der Gleichungen an speziellen Fällen zu überprüfen. Wir halten fest, dass die von der Oberflächenwelle mitgeführte Energie den Betrag

$$
-2 q * \frac{n^{\prime * *}}{\cos \alpha} E^{\prime} E^{\prime \prime}-2\left(C^{\prime \prime 2}+\tau_{1} \frac{\cos \beta}{\cos \alpha} D^{\prime \prime 2}+q \tau_{2} \frac{\cos \xi}{\cos \alpha} \mathrm{F}^{\prime \prime 2}\right)
$$

haben müsste.

Die Verifizierung der Energiegleichung an Hand des Musterfalles und fur die spezielle Annahme $\alpha=40^{\circ}$ geht von [17] aus, so dass

$$
\begin{aligned}
& J_{c}=0,18225 \\
& J_{d}=0,40824 \\
& J_{\mathrm{f}}=0,40950
\end{aligned}
$$

Zusammen

$$
=0.99999 \text {. }
$$


Da die einfallende Energie $=1$ gesetzt wurde, bleibt für die Energie der Grenzflächenwelle nur ein Betrag von 0,00001 übrig. In der Tat ist im Energieausdruck [22] für diese Welle der Wert des positiven Gliedes

$$
\begin{array}{r}
-2 q x \frac{w^{*}}{\cos \alpha} E^{\prime} E^{\prime \prime}=\left(q x \frac{w^{*}}{\cos \alpha}\right)\left(-2 E^{\prime} E^{\prime \prime}\right)=2,28384 \times(0,16253)= \\
=0,37120>0,
\end{array}
$$

wegen der entgegengesetzten Zeichen für $E^{\prime}$ und $E^{\prime \prime}$. Für die Berechnung der negativen Glieder hat man

$$
\begin{array}{rlrl}
-2 C^{\prime \prime 2}= & =-0,15982 \\
-2 \tau_{1} \frac{\cos \beta}{\cos \alpha} D^{\prime \prime 2}=-1,39972 \times 0,14136 & & =-0,19786 \\
-2 q \tau_{2} \frac{\cos \xi}{\cos \alpha} F^{\prime \prime 2}=-2,43500 \times 0,00555 & & =-0,01351 \\
\hline \text { Zusammen } & =-0,37119 .
\end{array}
$$

Für die Energie der geführten Welle bleibt also tatsächlich nur ein Betrag von 0,00001 Einheiten übrig.

Die Berechnung der Energie der geführten Welle ist mit jener Ungenauigkeit behaftet, mit der die aus fünfstelligen Taflen der trigonometrischen Funktionen behaftet sind. Diese Ungenauigkeit überträgt sich auf die Reflexions und Transmissionkoeffizienten. Verwendet man Computer mit einer grösseren Genauigkeit als fünf Stellen, so verkleinert sich entsprechend auch die berechnete Energie der geführten Welle. Man kann daraus nur den Schluss ziehen, dass diese Welle überhaupt keine Energie mit sich führt.

Auch das Verschwinden des imaginären Teiles der Energiegleichung [19] ist mit hinreichender Genauigkeit erfüllt. Man hat im Hinblick auf [15]

$$
\begin{array}{ccr}
C^{\prime} C^{\prime \prime}= & =0,09044 \\
\tau_{1} \frac{\cos \beta}{\cos \alpha} D^{\prime} D^{\prime \prime}=0,69986 \times 0,24996 & =0,17494 \\
q \tau_{2} \frac{\cos \xi}{\cos \alpha} F^{\prime} F^{\prime \prime}=1,21750 \times(-0,04284) & =-0,05216
\end{array}
$$

\begin{tabular}{lr}
\hline Summe & $=+0,21322$ \\
\hline $2 \times$ Summe & $=+0,42644$ \\
$q x \frac{u^{\prime *}}{\cos \alpha}\left(E^{\prime 2}-E^{\prime 2}\right)=2,28384 \times(-0,18672)$ & $=-0,42644$ \\
\hline Zusammen & $=0,00000$.
\end{tabular}


Das durchgerechnete Beispiel zeigt, dass die Energiegleichung in ihrer ursprünglichen Form auch weiterbesteht, wenn der Einfallswinkel der $P$-Welle den Mach' schen Winkel übersteigt.

4.

Bei weiterem Anwachsen des Einfallswinkels über $\alpha_{0}$ hinaus wird schliesslich ein Wert $\alpha=\alpha_{1}$ erreicht, bei dem auch die gebrochene $S$-Welle mit $\xi=90^{\circ}$ streifend austritt. Dieser Winkel ist der Grenzwinkel der totalen Reflexion für die sekundäre $S$-Welle. Im Grenzfall ist

$$
\begin{gathered}
\sin \xi=\frac{\gamma_{2}}{V_{1}} \sin \alpha=\tau_{2} \sin \alpha=1 \\
\sin \alpha_{1}==1 / \tau_{2}=V_{1} / \eta_{2}
\end{gathered}
$$

(in unserem Musterfall ist $\alpha_{1}=62^{\circ} 58^{\prime} 22^{\prime \prime}$ ). Auch hier transportiert die streifend austretende Welle keine Energie, wie ein Blick auf die Energiegleichung lehrt. Wegen $\cos \xi=0$ ist der dieser Welle entsprechende Energiebetrag $=0$.

Wegen $\cos \xi=\sqrt{1-\tau_{2} \sin ^{2}} \bar{\xi}=\sqrt{1-\tau_{2}^{2} \sin \alpha^{2}}$ wird bei weiteren Anwachsen von $\alpha$ über $\alpha_{1}$ hinaus $\tau_{2} \sin \alpha>1$, der $\cos \xi$ daher imaginär:

$$
\cos \dot{\xi}= \pm i i^{\prime} \tau_{2}^{2} \overline{\sin ^{2}} \overline{\alpha-1}= \pm i w_{1}^{*} \text {. }
$$

Auch hier besteht, ebenso wie in Nr. 2, Freiheit in der Vorzeichenwahl. Wir verwenden zunächst noch das + Zeichen, behalten uns aber vor, an späterer Stelle das physikalisch allein bedeutungsvolle Minuszeichen einzuführen.

Im System [5] zur Bestimmung der Amplitudenverhältnisse treten nun zwei weitere Glieder mit der imaginären Einheit auf:

$$
\begin{aligned}
& -\left(\frac{C}{A}\right)+\tau_{1} \operatorname{tg} \alpha\left(\frac{D}{A}\right)-i \frac{w^{*}}{\cos \alpha}\left(\frac{E}{A}\right)-\tau_{2} \operatorname{tg} \alpha\left(\frac{F}{A}\right)=-1 \\
& +\left(\frac{C}{A}\right)+\frac{\cos \beta}{\sin \alpha}\left(\frac{D}{A}\right)-\varkappa^{*}\left(\frac{E}{A}\right): \vdots \frac{w^{*}}{\sin \alpha}\left(\frac{F}{A}\right)=-1 \\
& -\left(\frac{C}{A}\right)+\tau_{1} \frac{\sin 2 \beta}{\cos 2 \beta}\left(\frac{D}{A}\right)+q x \frac{\cos 2 \eta}{\cos 2 \beta}\left(\frac{E}{A}\right)+2 i q \tau_{2}^{2} \frac{\sin 2 \xi}{\cos 2 \beta} w_{1}^{*}\left(\frac{F}{A}\right)=1 \\
& +\left(\frac{C}{A}\right)+\frac{1}{\tau_{1}} \frac{\cos 2 \beta}{\sin 2 \alpha}\left(\frac{D}{A}\right)+i q x^{2} \frac{w^{*}}{\sin 2 \alpha}\left(\frac{E}{A}\right)-q \frac{\tau_{2}}{\tau_{1}{ }^{2}} \frac{\cos 2 \xi}{\sin 2 \alpha}\left(\frac{F}{A}\right)=1
\end{aligned}
$$


Auch jetzt sind sämtliche Wurzeln des Systems komplex entsprechend [13]. Sie gehen bei Vertauschung des Vorzeichens der imaginären Einheit in ihre conjugierten Werte über.

Auch hier soll die allgemeine Auflösung des Systems [25] vermieden, dafür aber der Musterfall für $\alpha=65^{\circ}$ gerechnet werden. Mit diesem Wert befindet man sich deutlich jenseits des Grenzwinkels $\alpha_{1}$ der Totalreflexion für die transversale Sekundärwelle. Das System [25] lautet jetzt

$$
\begin{aligned}
& -\left(\frac{C}{A}\right)+1.23812\left(\frac{D}{A}\right)-3.43351 i\left(\frac{E}{A}\right)-2.40747\left(\frac{F}{A}\right)=-1 \\
& +\left(\frac{C}{A}\right)+0.94026\left(\frac{D}{A}\right)-1.94\left(\frac{E}{A}\right)+0.20697 i\left(\frac{F}{A}\right)=-1 \\
& -\left(\frac{C}{A}\right)+1.13814\left(\frac{D}{A}\right)-5.52075\left(\frac{E}{A}\right)+1.13661 i\left(\frac{F}{A}\right)=+1 \\
& +\left(\frac{C}{A}\right)+1.02287\left(\frac{D}{A}\right)+15.57816 i\left(\frac{B}{A}\right)+5.64698\left(\frac{F}{A}\right)=+1,
\end{aligned}
$$

und hat die Lösung

$$
\begin{aligned}
& \frac{C}{A}=-0,95063+0,24637 i \\
& \frac{D}{A}=-0,19632+0,19926 i \\
& \frac{E}{A}=-0,09109+0,24637 i \\
& \frac{F}{A}=+1,06065+0,20240 i .
\end{aligned}
$$

Die Energiegleichung wird ähnlich wie in Nr. 3 behandelt. Man hat in ihr nun $\cos \xi$ durch $i w_{1}^{*}$ zu ersetzen, so dass aus (4) nun wird 


$$
\begin{aligned}
& \left(C^{\prime}+i C^{\prime \prime}\right)^{2}+\tau_{1} \frac{\cos \beta}{\cos \alpha}\left(D^{\prime}+i D^{\prime \prime}\right)^{2}+ \\
& +i q x \frac{u^{*}}{\cos \alpha}\left(E^{\prime}+i E^{\prime \prime}\right)^{2}+i q \tau_{2} \frac{u^{\prime *}}{\cos \alpha}\left(F^{\prime}+i F^{\prime \prime}\right)=1 .
\end{aligned}
$$

Das Ordnen nach Real - und Imaginärteil fürt auf

$$
\begin{array}{r}
\left(C^{\prime 2}-C^{\prime \prime 2}\right)+\tau_{1} \frac{\cos \beta}{\cos \alpha}\left(D^{\prime 2}-D^{\prime \prime 2}\right)-2 q \times \frac{w^{*}}{\cos \alpha} E^{\prime} E^{\prime \prime}-2 q \tau_{2} \frac{w_{1}{ }^{7}}{\cos \alpha} F^{\prime} F^{\prime \prime}+ \\
+i\left\lfloor 2 C^{\prime \prime} C^{\prime \prime}+2 \tau_{1} \frac{\cos \beta}{\cos \alpha} D^{\prime} D^{\prime \prime}+q x \frac{w^{*}}{\cos \alpha}\left(E^{\prime 2}-E^{\prime \prime 2}\right)+q \tau_{2} \frac{w_{1}}{\cos \alpha}\right. \\
\left.\left(F^{\prime 2}-F^{\prime \prime}\right)\right]=1,
\end{array}
$$

$w_{0}$ nun der Imaginärteil für sich verschwinden muss. Die Restgleichunğ

$$
\begin{aligned}
\left(C^{\prime 2}-C^{\prime \prime 2}\right)+ & \tau_{1} \frac{\frac{n e}{2} r_{r}}{\cos \alpha}\left(D^{\prime 2}-D^{\prime \prime 2}\right)- \\
& -2 q \times \frac{w^{*}}{\cos \alpha} E^{\prime} E^{\prime \prime}-2 q \tau_{2} \frac{w_{1}^{*}}{\cos \alpha} F^{\prime} F^{\prime \prime}=1
\end{aligned}
$$

hat in dieser Form noch keine physikalische Bedeutung. Erst wenn man sie mit Hilfe des Ausdruckes

$$
2\left(C^{\prime \prime 2}+\tau_{1} \frac{\cos \beta}{\cos \alpha} D^{\prime \prime 2}\right)
$$

identisch umformt, lassen sich die einzelnen Glieder physikalisch deuten:

$$
\begin{gathered}
\left|\frac{C}{A}\right|^{2}+\tau_{1} \frac{\cos \beta}{\cos \alpha}\left|\frac{D}{A}\right|^{2}- \\
-2\left(C^{\prime \prime 2}+\tau_{1} \frac{\cos \beta}{\cos \alpha} D^{\prime \prime 2}\right)+q x \frac{u^{\text {* }}}{\cos \alpha} E^{\prime} E^{\prime \prime}+q \tau_{2} \frac{w_{1}^{*}}{\cos \alpha} F^{\prime} F^{\prime \prime}=1 .
\end{gathered}
$$

Es ist dann nämlich 
E. HARDTWIG

$\left|\frac{C}{A}\right|^{2} \ldots$ die Energie $J_{c}$ der reflektierten $P$-Welle $\tau_{1} \frac{\cos \beta}{\cos \alpha}\left|\frac{D}{A}\right|^{2} \ldots$ die Energie $J_{d}$ der reflektierten $S$-Welle

und für den Ausdruck in der Klammer bleibt dann die Deutung als Energie der beiden geführten Wellen zusammengenommen $J_{c}+J_{f}$. Es ist aber nich möglich, sie in einen Anteil $J_{e}$ und einen Anteil $f_{f}$ aufzuspalten. Der Klammerausdruck ist mit einem Minuszeichen versehen. Eines der beiden Produkte $E^{\prime} E^{\prime \prime}$ oder $F^{\prime} F^{\prime \prime}$ ist aber immer negativ, das mit ihm behaftete Glied also positiv und, wie sich zeigt, von solch hohem Betrag, dass es die übrigen zusammengenommen an Grösse übertrifft. Tatsächlich bekäme man also einen positiven Wert für die von den geführten Wellen transportierte Energie, wenn diese nicht überhaupt $=0$ ist. Gerechnete Beispiele lassen dies mindestens vermuten.

Die C'berpriilung der Energiegleichung soll an Hand des Musterfalles für $\alpha=65^{6}$ vorgenommen werden. Aus [13] und [27] entnimmt man

$$
\begin{aligned}
C^{\prime 2} & =0,00521 & D^{\prime 2}=0,03970 \\
\left|\frac{C^{2}}{A}\right|^{2} & =0,90890 & \left|\frac{D}{A}\right|^{02}=0,07825 \\
E^{\prime} E^{\prime \prime} & =-0,02244 & F^{\prime} F^{\prime \prime}=+0,21468
\end{aligned}
$$

und daher

$$
\begin{aligned}
& \left|\frac{C}{A}\right|^{2} \cdots=J_{c}= \\
& \tau_{1} \frac{\cos \beta}{\cos \alpha}\left|\frac{D}{A}\right|^{2}=J_{d}=1,16417 \times 0,07825
\end{aligned}
$$$$
==0,09109
$$

Energie der beiden reflektierten

Wellen zusammengenommen

Formal is dies wieder fast die gesamte, eingestrahlte Energie. Zur Berechnung der Energie der geführten Wcller hat man 
$q \varkappa \frac{w^{*}}{\cos \alpha} E^{\prime} E^{\prime \prime}=8,01153 \times(-0,02244)$

$=-0,17978$

$\tau_{1} \frac{\cos \beta}{\cos \alpha} D^{\prime 2}=1,16417 \times 0,03970$

$=0,04622$

$q \tau_{2} \frac{13_{i}^{*}}{\cos \alpha} F^{\prime} F^{\prime \prime}=0,59792 \times 0,21468$

$=0,12836$

Energie des mitgeführten Wellenpaares

$=0,00001$.

Man hat es ersichtlich auch hier mit einer Wellenhaut zu tun. Die Einheit in der fünften Stelle rührt offenkundig von Rundungsfehlern her.

In diesen Zusammenhang soll auf folgendes Hingewiesen werden: Dort, wo die Lösungen des Linearsystems [5] infolge Imaginärwerdens von Koeffizienten selbst komp'exe Zahlen werden, treten an der Unstetigkeitsfläche Phasenverschiebungen auf. Die reflektierten bzw. gebrochenen Sekündärwellen haben eine andere Phase als die einfallende Welle. Dies wird hier nicht näher ausgeführt.

Ferner: Den hier besprochenen Sekundärwellen überlagern sich immer die zu den beiden Machschen Winkeln gehörenden Refraktions -und Mintropwellen (Kopfwellen). Das von den Geophonen aufgenommene We!lenbild ist also wesentlich komplizierter, als es hier den Anschein hat. Für den hier zu verfolgenden Zweck ist dies aber ohne Belang.

5.

Verlauf der Reflexions - und Transmissionskoeffizienten als Funk. tionen der Einfallswinkel zwischen 0 und 90 Grad.

Man darf, da das Eintreten der Totalreflexion bzw. das Einsetzen von Grenzschichtwellen physikalisch bemerkenswerte Vorgänge sind, erwarten, dass sie sich in Auffälliger Weise im Verlaufen der Reflexions und Transmissionskoeffizienten kundtun. In der Sprengseismik beobachtbar und praktisch erkennbar sind nur die Reflexionskoeffizienten, da nur die reflektierten Sekundärwellen an die Erdoberfläche zurückkommen.

Bei wachsendem Einfallswinkel $\alpha$ wird die gebrochene $P$-Welle 
von dem Augenblick an, da $\alpha$ den ersten Machschen Winkel erreicht, streifend austreten und, wenn überhaupt, ein Minimum an Energie mitführen. Man darf dann annehmen, dass die restlichen Sekündärwellen in auffallender Weise ein Mehr an Energie transportieren - dies aber müsste sich in entsprechender Zunahme der Amplituden äussern. Leider ist die Energie einer ankommenden Welle nicht unmittelbar beobachtbar. Was der Beobachtung in erster Linie zugänglich ist sind die Amplituden bzw. die Amplituden-verhältnisse, praktisch also die Reflexionskoeffizienten. Allerdings brauchen diese nicht der oben angedeuteten Überlegung $\mathrm{zu}$ folgen. Trotzdem kann gesagt werden, dass die Reflexionskoeffizienten in sehr empfindlicher Weise auf Vorgünge wie etwva Totalreflexion oder Änderung in den physikalischen Konstanten reagieren.

Um die Art und Weise dieses Reagierens kennen zu lernen, wurde an Hand des Musterfalles Gl. [9] das Verhalten der Reflexionskoeffizienten $C / A, D / A$ und der Transmissionskoeffizienten $E / A$ und $F / A$ als Funktionen des Einfallswinkels untersuch. Wie bereits erwähnt, sind hier die Grenzwinkel der Totalreflexion $\alpha_{0}=30^{\circ} 57^{\prime} 02^{\prime \prime}$ für die $P$-Welle und $\alpha_{1}=62^{\circ} 58^{\prime} 22^{\prime \prime}$ für die $S$-Welle. Nach ihnen muss sich die Auswahl jener Werte $\alpha$ richten für die diese Koeffizienten berechnet werden sollen. Tatsächlich wurden sie berechnet für

$$
\begin{gathered}
\alpha=0^{0}, 10^{\circ}, 20^{\circ}, 30^{\circ}, 30^{\circ} 57^{\prime} 02^{\prime \prime}, 32^{\circ}, 35^{\circ}, 40^{\circ}, \\
50^{\circ}, 60^{\circ}, 62^{0} 58^{\prime} 22^{\prime \prime}, 64^{0}, 65^{\circ}, 70^{\circ}, 80^{\circ} .
\end{gathered}
$$

Um speziell in der Nähe der Grenzwinkel der Totalreflexion das Verhalten der $C / A, \ldots$ genauer kennen $z u$ lernen, müssten in der Umgebung dieser Stellen noch weitere Punkte angenommen werden. Für jeden dieser - Werte wurde das System [5] gebildet und streng gelöst. Die trigonometrischen Funktionen sind etwa auf 5 Stellen genau, entsprechend is die Genauigkeit in den Lösungen. Man muss also mit Unsicherheiten in den letzten 1 bis 2 Dezimalen rechnen. Für die Quadratwurzeln $w^{*}$ und $w_{1}^{*}$ wurde jeweils das negative Zeichen gewählt, um physikalisch brauchbare Ausdrücke für die (hier nicht behandeltc) Teilchenverschiebungen zu erhalten. Das Ergebnis der Rechnung ist in Tabelle II zusammengefasst.

Die Amplituden bzw. Amplitudenverhältnisse werden, wie aus dem Vorangehenden zu ersehen, durch die Beträge der komplexen Zahlen gemessen. Fraglich kann nur das Vorzeichen der jeweiligen Wurzel sein. 


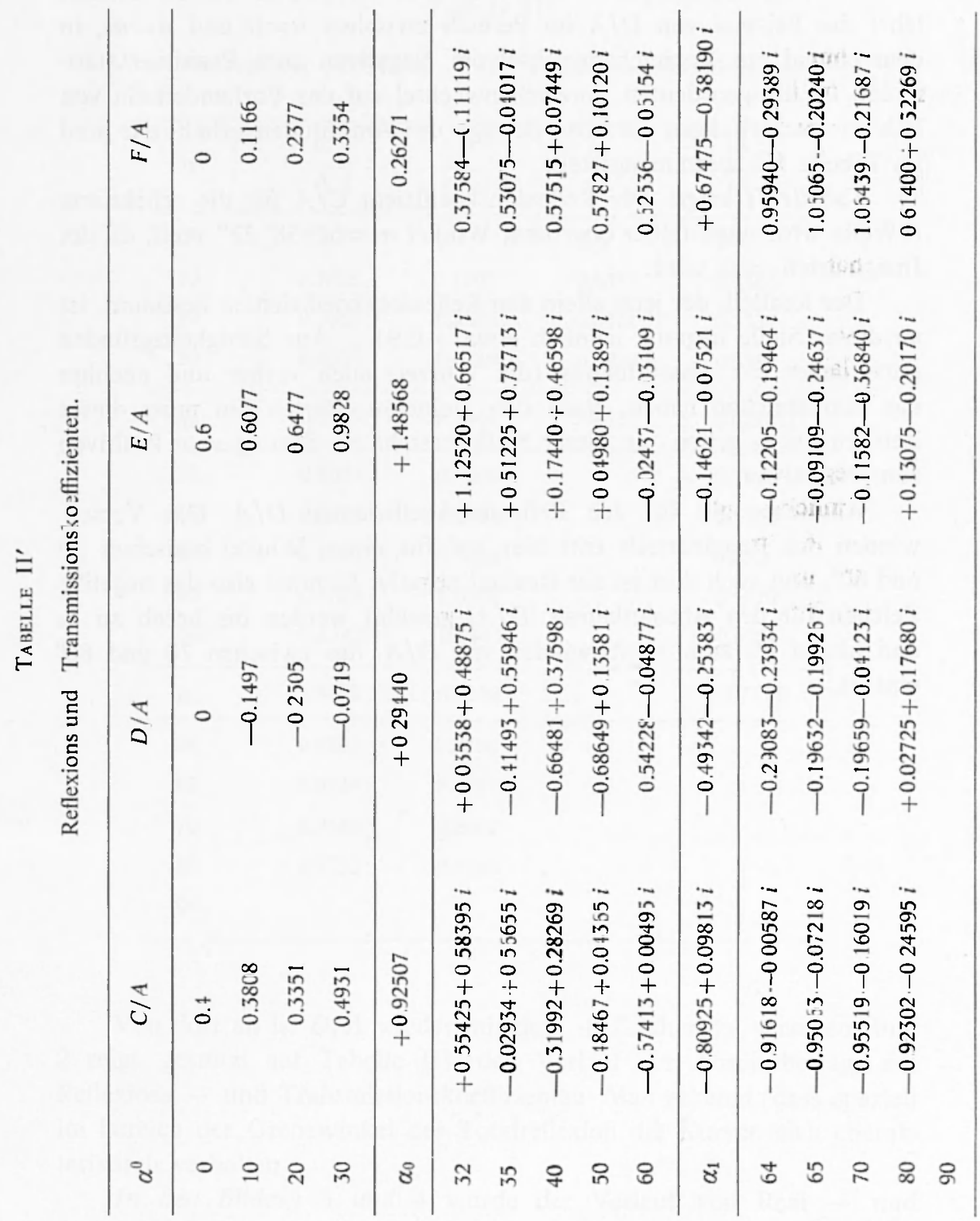


Dass Reflexionskoeffizienten auch negatives Vorzeichen haben können lehrt das Beispiel von $D / A$ im Bereich zwischen $\alpha=0$ und $\alpha=\alpha_{0}$, in dem einmal ein Vorzeichenwechselvom Negativen zum Positiven stattfindet. Im übrigen deuten Vorzeichenwechsel auf das Vorhandensein von Pahsenverschiebungen hin. Die Beträge der Amplitudenverhältnisse sind im Tabelle III zusammengestellt.

Tabelle II zeigt: Der Reflexionskoeffizient $C / A$ für die reflektierte $P$-Welle wird unmittelbar ober dem Winkel $\alpha_{1}=62^{\circ} 58^{\prime} 22^{\prime \prime}$ reell, da der Imaginärteil $=0$ wird.

Der Realteil, der jetzt allein den Reflexionskoeffizienten bestimmt, ist an dieser Stelle negativ, nämlich etwa -0.91 ... Aus Stetigkeitsgründen muss daher der Absolutbetrag (die Wurzel) auch vorher und nachher das Minuszeichen haben. Nach den kleineren Werten hin muss dieses Zeichen bis $\alpha_{0}$ gelten - an dieser Stelle besteht ein Sprung vom Positiven zum Negativen.

Ähnliches gilt für den Reflexionskoeffizienten $D / A$. Das Verschwinden des Imaginärteils tritt hier auf für einen Winkel zwischen 50 und $60^{\circ}$, und auch dort ist der Realteil negativ. Es muss also das negative Zeichen für den Absolutbetrag $|D / A|$ gewählt werden bis herab $\mathrm{zu} \alpha_{0}$ und hinauf bis zum Verschwinden von $D / A$, das zwischen 70 und $80^{\circ}$ eintritt. 
TABELLE III

Absolutbeträge der Reflexions - und Transmissionskoeffizienten.

\begin{tabular}{lllll}
\hline$\alpha$ & $\left|\frac{C}{A}\right|$ & $\left|\frac{E}{A}\right|$ & $\left|\frac{F}{A}\right|$ & $\left|\frac{F}{A}\right|$ \\
\hline $0^{0}$ & 0.4 & 0 & 0.6 & 0 \\
10 & 0.3808 & 0.1497 & 0.6077 & 0.1166 \\
20 & 0.3351 & 0.2505 & 0.6477 & 0.2377 \\
30 & 0.4931 & 0.0719 & 0.9828 & 0.3354 \\
\hline$\alpha_{0}$ & $(0.9251)$ & 0.2944 & 1.4857 & $(0.2627)$ \\
\hline 32 & 0.8051 & 0.4900 & & 0.3919 \\
35 & 0.5673 & 0.6965 & & 0.5323 \\
40 & 0.4269 & 0.7638 & & 0.5800 \\
50 & 0.4866 & 0.6998 & & 0.5879 \\
60 & 0.6742 & 0.5445 & & 0.6284 \\
\hline$\alpha_{1}$ & 0.8152 & 0.5548 & & \\
\hline 64 & 0.9162 & 0.3766 & & \\
65 & 0.9534 & 0.2797 & & \\
70 & 0.9685 & 0.2007 & & \\
80 & 0.9552 & 0.1789 & & \\
90 & & & & \\
\hline
\end{tabular}

Von dort an ist $D / A$ wieder mit dem + Zeichen zu versehen. Bild 2 zeigt, gestützt auf Tabelle III, den Verlauf der Absolutbeträge der Reflexions - und Transmissionskoeffizienten. Man erkennt, dass speziell im Bereich der Grenzwinkel der Totalreflexion die Kurven sich charakteristisch verhalten.

In den Bildern 3 und 4 wurde der Verlauf von Real - und Imaginärteil von $C / A$ bzw. $D / A$ als Funktionen des Einfallswinkels dargestellt. Die beiden Diagramme zeigen folgendes: 


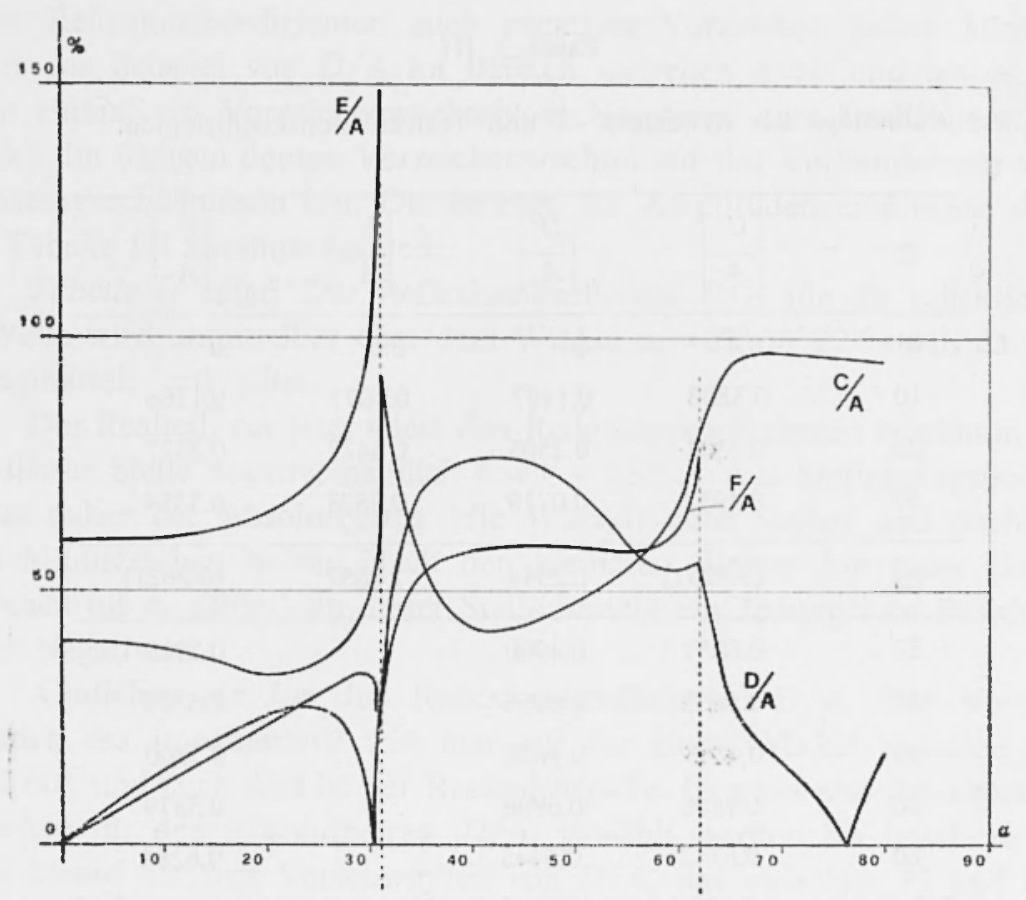

Bild 2 - Verlauf der Absolutbeträge der Reflexionskoeffizienten $C / A$ und $D / A$ sowie der Transmissionskoeffizienten $E / A$ und $F / A$ als Funktionen des Einfallswinkels $\alpha$.

a) Beginn des totalen Reflexion für die Verdichtungswelle.

b) Beginn des totale Reflexion für die Scherungswelle.

Bild 3 (Verlauf für $C / A$ ): Wenn der Einfallswinkel $\alpha$ sich wachsend dem Grenzwinkel der Totalreflexion nähert, beginnt der (hier reelle) Wert von $C / A$ ausserordentlich stark $\mathrm{zu}$ steigen und nach Passieren der kritischen Stelle ebenso rasch- jetzt als Realteil- zu fallen. Schon bei weniger als $35^{\circ}$ ist der Nullpunkt erreicht, von da an bleibt er negativ. Auf die zweite Totalreflexion spricht er nicht deutlich an, zumindest nicht an der kritischen Stelle.

Der Imaginärteil, der bis $\alpha=\alpha_{0}$ gleich Null war, fällt kurz danach ausserordentlich steil $\cdot a b$, nimmt weiter fast bis Null zu und reagieri auf die zweite Totalreflexion mit einem scharfen, aber nicht konformen Abstiegsbuckel. 


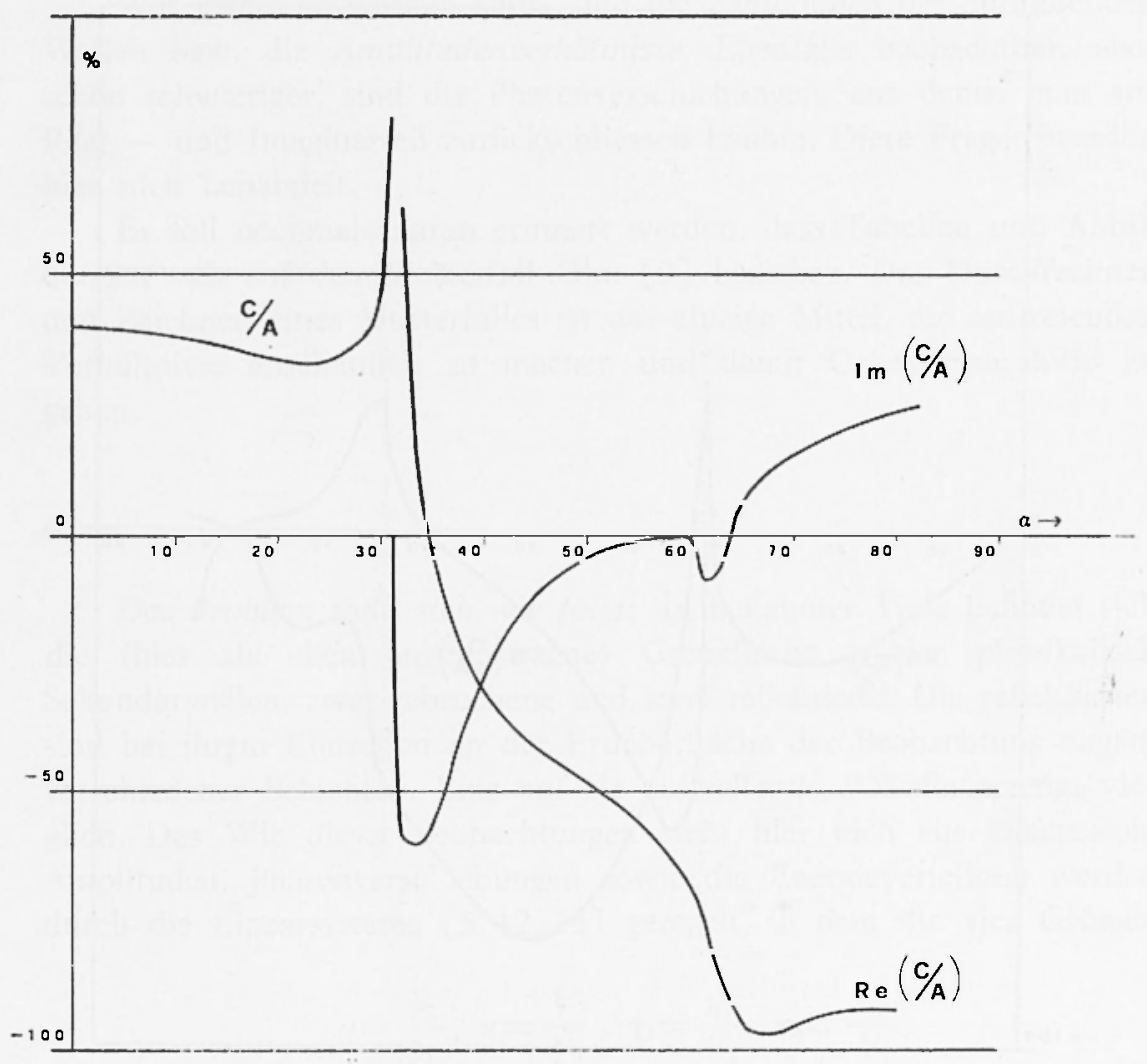

Bild 3 -Realteil $\operatorname{Re}(C / A)$ und Imaginärteil $\mathrm{Jm}(C / A)$ des Reflexionskoeffizienten $C / A$ des sekundären Verdichtungswelle als Funktionen des Einfallswinkels $\alpha$

Bild 4 (Verlauf für $D / A$ ): Der zunächst negative Wert von $D / A$ beginnt bei Annäherung von $\alpha$ an den Grenzwinkel der Totalreflexion $\alpha_{0}$ erst langsam, dann stark zu steigen, passiert kurz vor $\alpha_{0}$ die Nullstelle und steigt reell-positiv an bis zum Argumentwert $\alpha_{0}$, wo der Maximalwert von $D A$ erreicht wird. Nach Passieren der kritischen Stelle sinkt der Realteil rasch $a b$, passiert kurz darauf die Nullstelle in umgekehrter Richtung und bleibt negativ bis gegen $\alpha=80^{\circ}$. Auf die zweite Totalreflexion spricht der Realteil nich merklich an.

Der Imaginärteil bleibt bis $\alpha=\alpha_{0}$ gleich Null, steigt bei $\alpha_{0}$ sprunghalt auf einen negativen Wert an, um kurz darauf wieder abzuklingen und bei etwa $58^{\circ}$ die Nullstelle zu passieren. Auf die zweite 


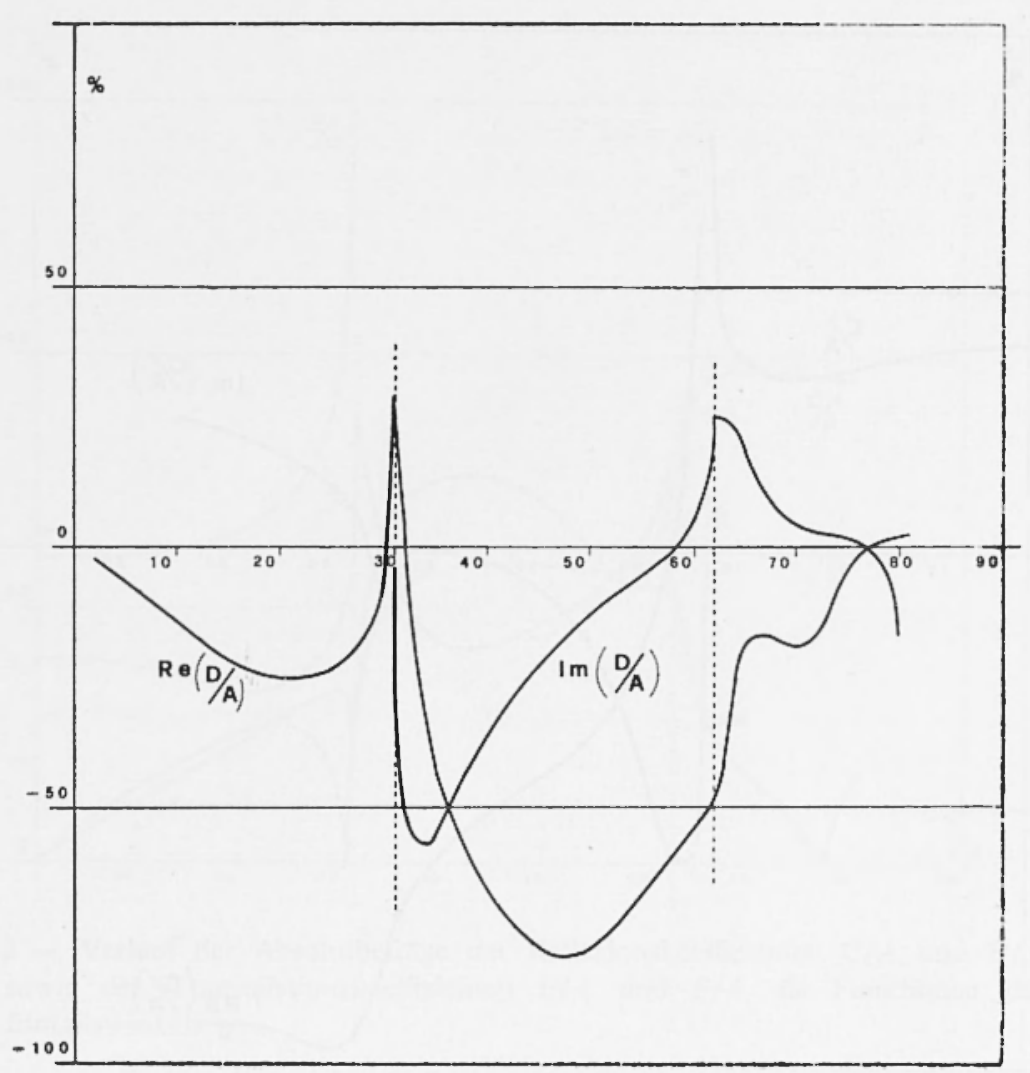

Bild 4-Realteil $\operatorname{Re}(D / A)$ und Imaginärteil $\mathrm{Jm}(D / A)$ des Reflexionskoeffizienten $D / A$ des sekundären Scheringswelle als Funktionen des Einfallswinkels $\alpha$.

Totalreflexion reagiert der Imaginärteil mit einer markanten, hornartigen Ausbuchtung.

Wäre bei einer Sprengung im Gelände jeweils von den reflektierten Wellen Realteil für sich und Imaginärteil für sich beobachtbar, so könnte man aus dem Verhalten dieser beiden Teile die beiden Grenzwinkel der Totalreflexion feststellen und aus ihnen auf die Materialkonstanten schliessen. Auch die Lage der Nullstellen könnte man zur Bestimmung der Materialkonstanten heranziehen. Leider sind aber die komplexen Lösungen $C^{\prime}+C^{\prime \prime}, D^{\prime}+D^{\prime \prime} \ldots$ der Systeme $[12,25]$ zunächst nur Rechengrössen, die der Beobachtung nicht zugängig sind, zumindest nicht unmittelbar. 
Was gemessen werden kann, sind die Amplituden der eintreffenden Wellen bzw. die Amplitudenverhältnisse. Ebenfalls beobachtbar, aber schon schwieriger, sind die Phasenverschiebungen, aus denen man auf Real - und Imaginärteil zurückschliessen könnte. Diese Fragen werden hier nich behandelt.

Es soll nochmals daran erinnert werden, dass Tabellen und Abbildungen sici auf den Musterfall Gln. [9] beziehen. Das Durchrechnen und Zeichnen eines Musterfalles ist das einzige Mittel, die auftretenden Verhältnisse anschaulich zu machen und damit Orientierungshilfe zu geben.

6.

Das Problem stellt sich wie folgt: In bekannter Tiefe befindet sich die (hier als eben angenommene) Grenzfläche zweier physikalisch Sekundärwellen, zwei gebrochene und zwei reflektierte. Die reflektierten sind bei ihrem Eintreffen an der Erdoberfläche der Beobachtung zugänverschiedener Schichten. Eine auf sie auftreffende $P$-Welle erzeugt vier glich. Das Wie dieser Beobachtungen steht hier nich zur Diskussion. Amplituden, Phasenverschiebungen sowie die Energieverteilung werden durch die Linearsysteme $[5,12,25]$ geregelt, ir dem die vier Grössen

$$
q=\frac{\rho_{1}}{\rho_{1}}, \quad x=\frac{V_{2}}{V_{1}}, \tau_{1}=\frac{\eta_{1}}{V_{1}}, \tau_{2}=\frac{\eta_{1}}{V_{1}}
$$

Diesem Umstand wurde durch Erstellen der Tabelle III für den Musterfall Rechnung getragen. Um vollständig zu sein, wurden auch die Beträge $|E / A|$ und $|F / A|$ in die Tabelle aufgenommen. Da bei Erreichen von $\alpha_{0}$ die $e$-Welle, bei Erreichen von $\alpha_{1}$ die $f$-Welle zu bestehen aufhören, endet bei diesen Einfallswinkeln die betreffende Wertereihe. Zusätzlich wurde der Verlauf der vier Beträge $C / A, \ldots$ graphisch dargestellt (Bild 2).

Es bedeutet keinen Wirderspruch, dass in Tabelle III die Transmissionskoeflizienten bei den Machschen Winkeln aufhören, in Tabelle II aber die Lösungen der entsprechenden Lineargleichungen für den ganzen Bereich $0<0<90^{\circ}$ angeführt sind. Solche Lösungen existieren für den ganzen Bereich, nur tragen sie jenseits der entsprechenden Machschen Winkel zur Wellenhaut entlang der Trennungsiläche bei, deren Energietransport zumindest vernachlässigbar klein ist. 
Die Betrachtung des Verlaufs der Reflexionskoeffizienten als Funktionen von $\alpha$ lässt folgendes erkennen:

a) Reflexionskoeffizient $C / A$ der skundüren P-Welle: Die Grösse $C / A$ spricht ausserordentlich scharf auf die erste Totalreflexion an, nicht aber auf die zweite.

Aus ihrem Verlaul müsste sich der Grenzwinkel $\alpha_{0}$ und damit die Materialkonstente $x$ mit grosser Genauigkeit bestimmen lassen. Hingegen wird die zweite Totalreflexion (bei $\alpha_{1}$ ) von $D / A$ sozusagen nicht zur Kenntnis genommen. Hier scheint sich ein gewisser Antagonismus abzuzeichnen: die jeweilige Totalreflexion beeinflusst in erster Linie die $P$-Wellen, gleichgiltig ob die reflektierten oder gebrochenen, bevorzugt $P$-Welle behandelt. Von vornherein darf also vermutet werden, dass die $P$-We!len, gleigiltig ob die reflektierten oder gebrochenen, bevorzugt sein werden. Das ist in der Tat auch der Fall - dazu gehört auch, dass gerade $C / A$ auf die erste Totalreflexion so scharf anspricht. Das hier angedeutete Prinzip findet übrigens eine Bestätigung bei der Betrachtung des Verlaufs von $E / A$ und $F / A$ (welche beide nicht beobachtet werden können): $E / A$ spricht auf $\alpha_{0}$ an (P-Welle !), $F / A$ hingegen auf $\alpha_{1}(S$ Welle!).

Das beschriebene Verhalten findet in der Optik (die man ja auch mit Hilfe der Elastizitätstheorie behandeln kann) ein gewisses Analogon. Dort reagiert der Reflexionskoeffizient (es gibt nur einen) auf den Grenzwinkel der Totalreflexion derart scharf, dass sich dieser und damit der Brechungsindex $n$ (hier $x$ ) mit grosser Genauigkeit bestimmen lässt (Methode von Kchlrausch).

Auch die Nullstellen von D/A lassen sich mit grosser Genauigkett leststellen. Die Bedingungen für das Verschwinden der Reflexionskoeffizienten - also für das Vorhandensein von Nullstellen - wurden hier nicht formuliert, vor allem nicht, weil sie recht kompliziert ausfallen würden. In sie würden, neben den trigonometrischen Funktionen von $\alpha$, die vier Materialkonstanten eingehen. Die Bedingungen würden also, für die jeweilige Nullstelle formuliert, Bedingungen für die Materialkonstanten liefern, also ihre Berechnung möglich machen.

Weitere markante Punkte sind die Maxima und Minima für $C / \Lambda$ und $D / A$, wenngleich diese Punkte nicht so scharf definiert sind. Die Bedingungen für ihr Eintreten lassen sich ebenfalls, indem sie für die jeweiligen Extremstellen formuliert werden, ails Gleichungen zwischen den Materialkonstanten auffassen und zu ihrer Berechnung heranziehen. 
Im ganzen Bereich der möglichen Einfallswinkel ist der Verlauf der beiden Reflexionskoeflizienten $C / A$ und $D ! A$ so ahwechslungsreich, dass den vier Materialkonstanten i. A. mehr als vier Beziehungen gegenüberstehen werden, zumal ja in der Nähe kritischer Stellen eine Häufung von Beobachtungsstationen wird vorgesehen werden müssen. Hier kann dann nur die Methode der kleinsten Quadrate zur Bestimmung $\mathrm{der}$ "plausibelsten Werte» für die Materialkonstanten $q, x, \tau_{\mathbf{1}}, \tau_{2}$ hcrangezcgen werden, wobei die weniger genauen Beobachtungen mit geringerem Gewicht zu versehen sind. Unter der Voraussetzung, dass die Materiallkonstanten $p_{1}, V_{1}, \eta_{1}$ der oberen Schicht bekannt sind (was meist der Fall ist) können dann aus $q, x, \tau_{1}, \tau_{2}$ die Konstanten $p_{2}, V_{2}, \tau_{2}$ bestimmt werden. Für $\eta_{1} / V_{1}$ ergibt sich dann ein plausibelster Wert.

Es ist klar, dass alle hier vorgebrachten Überlegungen auf den Fall übertragen werden können, dass die einfallende Welle eine $S$-Welle ist. Da bei jedem Schuss neben den Dilatationswellen auch Scherungswellen cntstehen, müsstein auch diese Betrachtungen - und sei es auch nur zur Kontrolle - angestellt werden.

In jedem Fall ist die Lage so, dass aus dem Verlauf der Reflexionskceffizienten alles bestimmi werden kann, was zu bestimmen durch die Reflexionsmethode ïberhaupt möglich ist.

Auf das hier skizzierte Verfahren, Aussagen über gewisse, sonst nich zugängliche Materialkonstanten zu machen scheint noch nicht aufmerksam gemacht worden zu sein. Seine praktische Verwertbarkeit hängt von messtechnischen Umständen ab. Das Feststellen von Reflexionen ( $P$-und $S$-Welle) an der Erdoberfläche bereitet keine Schwierigkeiten. Solche erheben sich erst, wenn die Amplituden bestimmt werden sollen, mit denen die Reflexionen ankommen. Diese sind bekanntlich durch das Auftreten der so unerwünschten Oberflächenwellen (Roller) gestört, die erst weganalysiert werden müssen. Zum Glück ist das moglich. Auch kcmmt es nicht so sehr auf die Werte der Amplituden an als auf ihr Verhalten als Funktionen ven $\alpha$, bzw. auf ihr Verhältnis zu einer willkürlich vorgegeben Amplitude.

Praktisch müsste so vorgegangen werden. dass Geophone entlang einer Geraden aufgestellt werden, die vom Sprengpunkt bzw. Bohrloch ausgcht. Wie bereits erwähnt müssten die Geophone dort, wo direkte Totalreflexionen oder scnstige kritische Punkte zu erwarten sind, etwas dichter angeordnet werden. Bild 5 zeigt die grundsätzliche Anordnung.

Bei gegebener Tiefe des Sprengpunktes, gegebener Tiefe der 


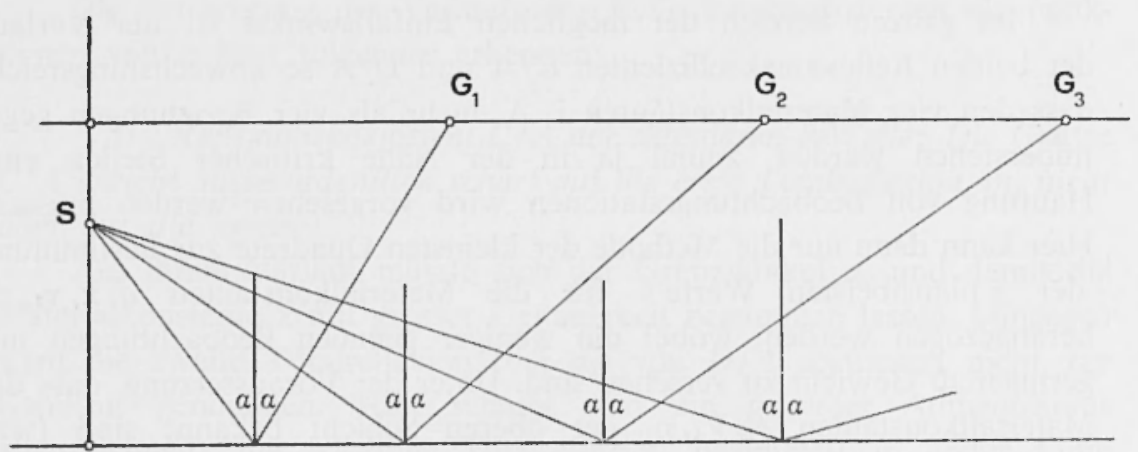

Fig. 5.

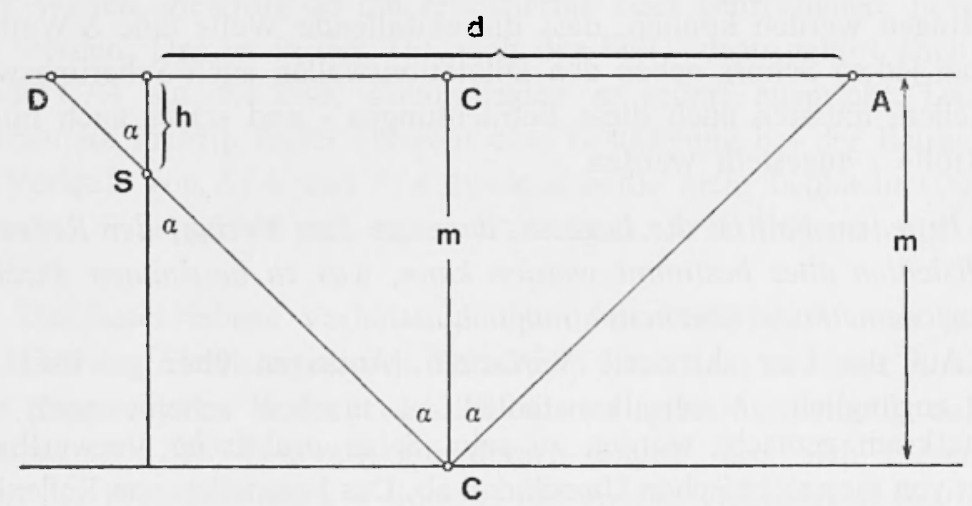

Fig. 6.

Unstetigkeitsfiäche und gegebenem Abstand des Geophons von der Mündung des Bohrloches ist der Winkel $\alpha$ errechenbar.

Es soll bedeuten:

$h=$ Tiefe des Sprengpunktes

$m=$ Schichtmächtigkeit (Tiefe der Unstetigkeitsfläche)

$d=$ Abstand Geophon-Bohrlochmündung.

Dann ist im gleichschenkligen Dreieck $A D C$ die Basis $d+x$ mit $x=h . \operatorname{tg} \alpha$. 
Aus dem rechtwinklingen Dreieck $A C C$ folgt dann

$$
\operatorname{tg} \alpha=\frac{A C}{m}=\frac{x+d}{2 m}-\frac{h \cdot \operatorname{tg} \alpha+d}{2 m}
$$

Somit ist

$$
\operatorname{tg} \alpha=\frac{d}{2 m-h}
$$

Der Verlauf der Funktionen $C / A$ und $D / A$ kann also punktweise ermittelt werden.

7.

Physikalisch ist die Verteilung der Energie auf die vier Sekundärwellen wichtiger als es die Reflexions - und Transmissionskoeffizienten sind. Leider ist die Energiemenge, die jeweils mit einer Sekundärwellen an der Erdoberfläche ankommt, nich unmittebar messbar. Der Messung zugänglich sind allein die Amplituden und Perioden.

Es lohnt vielleicht trotzdem, diese Energiemengen zu bestimmen, natürlich auf rechnerischem Wege, wobei die Reflexions - und Trans missionskoeffizienten als bekannt vorausgesetzt werden. Die Energieverteilung variiert mit dem Einfallswinkel $\alpha$ der Primärwelle, es ist daher

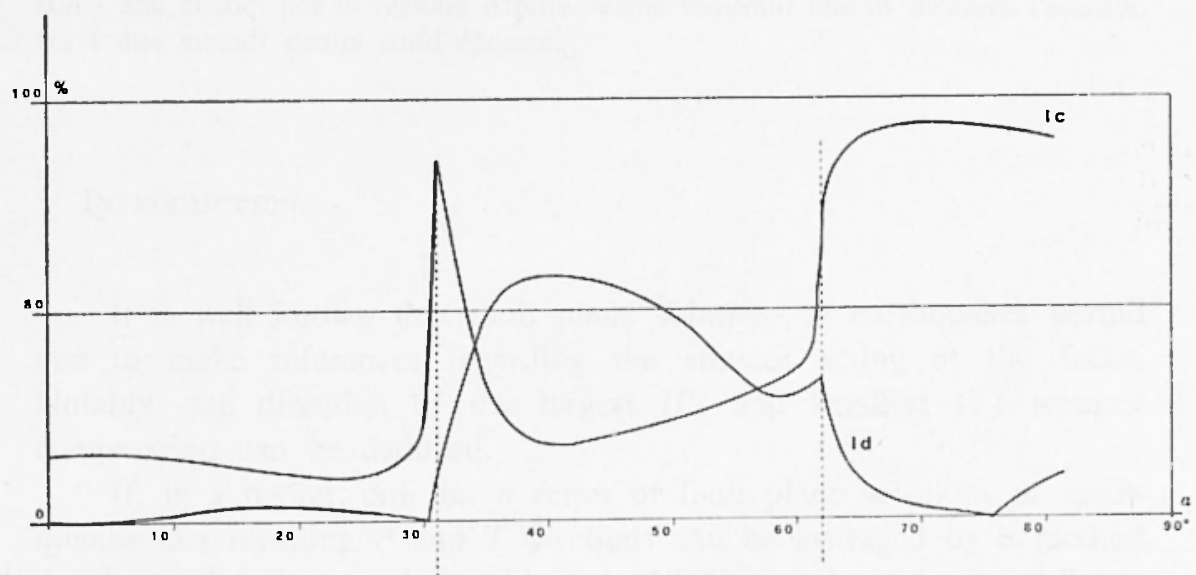

Bild 7 - Verlauf der aüf die beiden reflektierten Wellen entfallenden Energiemengen $\mathrm{J}_{\mathrm{c}}$ und $\mathrm{J}_{\mathrm{d}}$ als Funktionen des Einfallswinkels $\alpha$. 
zweckmässig, die Energiemengen als Funktionen von $\alpha$ darzustellen. Dabei ist von den Energiegleichungen [4], [18], [29] auszugehen. Hier wurden nur die Energien $J_{c}$ und $J_{d}$ für den Musterfall berechnet und als Funktionen von $\alpha$ dargestellt. Die Berechnung von $J_{e}$ und $J_{f}$ unterblieb

$\mathrm{Da}$ in den jeweiligen Ausdruck für die Energie das Quadrat der Amplitude eingeht ist es nich zu verwundern, dass die Energien dem Gang der Amplituden im Grossen und Ganzen folgen. Kritisch ist die Energieverteilung im Bereich der Machschen Winkel. Das Einsetzen der Totalreflexion veründert das Bild vollständig, Refraktionswelle und Mintropwelle (Kopfwelle) werden weder vom Linearsystem [5] noch von der zugehörigen Energiegleichung beschrieben. Hier hat die integrale Behandlung des Reflexions - und Brechungsverhaltens einzusetzen. 